\title{
Shell model estimate of electric dipole moments in medium and heavy nuclei
}

\author{
E. Teruya ${ }^{1, a}$, N. Yoshinaga ${ }^{1}$, and K. Higashiyama ${ }^{2}$ \\ ${ }^{1}$ Department of Physics, Saitama University, Saitama City 338-8570, Japan \\ ${ }^{2}$ Department of Physics, Chiba Institute of Technology, Narashino, Chiba 275-0023, Japan
}

\begin{abstract}
It is evidence for an extension of the Standard Model in particle physics, if static electric dipole moments (EDMs) are measured for any elementary particle. The nuclear EDM arises mainly from two sources: one comes from asymmetric charge distribution in a nucleus and the other is due to the nucleon intrinsic EDM. We estimate the nuclear EDMs from two sources for the $1 / 2_{1}^{+}$states in Xe isotopes by a shell model approach using full orbitals between magic numbers 50 and 82 .
\end{abstract}

\section{Introduction}

The Standard Model in particle physics violates time reversal $(T)$ invariance, but only through a single phase in the Cabibbo-Kobayashi-Maskawa matrix that mixes quark flavors. The resulting $T$ violation is therefore very weak, and static electric dipole moments (EDMs) of nucleons, electrons, or atoms are predicted to be too small to be observed [1]. For the neutron EDM, $\left|d_{N}\right|<2.9 \times 10^{-26} e \mathrm{~cm}$ was measured by Baker et al. [2], in contrast to a theoretical estimate of $\left|d_{N}\right| \sim 10^{-32} e \mathrm{~cm}$ [3] by the Standard Model. Some of the tightest constrains on $T$ violation come from atomic EDM experiments. In ${ }^{129} \mathrm{Xe}$ and ${ }^{199} \mathrm{Hg}$, upper limits of atomic EDMs were measured: $\left|d\left({ }^{129} \mathrm{Xe}\right)\right|<4.1 \times 10^{-27} e \mathrm{~cm}[4]$ and $\left|d\left({ }^{199} \mathrm{Hg}\right)\right|<3.1 \times 10^{-29} e \mathrm{~cm}[5]$.

Recently, it was reported that the nuclear EDM would be measured directly by using an ionic atom instead of a neutral atom [6,7]. The nuclear EDM is induced mainly by two different sources of mechanism. One comes from two-body nuclear interactions violating parity $(P)$ and $T$ invariance. The other comes from the intrinsic nucleon EDM. In this work we calculate the nuclear EDMs for the $1 / 2_{1}^{+}$states in Xe isotopes from two different sources. Nuclear EDMs are calculated using wave functions obtained by the pair-truncated shell model (PTSM) [8]. The upper limit of the nuclear EDM is estimated only for the ${ }^{129} \mathrm{Xe}$ ground state (spin and parity $1 / 2^{+}$) since in other Xe nuclei the ground states have spin and parity $3 / 2^{+}$. We do not discuss other isotopes in this work, but similar results are obtained for Ba isotopes. Their results will be presented in a forthcoming paper [9].

\section{Theoretical framework}

In the PTSM we restrict the full shell model space to a subspace of collective pairs. The building blocks of this model consist of the angular momenta zero $(S)$ and two $(D)$ collective pairs. The many-

\footnotetext{
ae-mail: teruya@nuclei.th.phy.saitama-u.ac.jp
} 
body wave functions of even-nucleon systems for neutrons or protons are created by applying the pair creation operators $\hat{S}^{\dagger}$ and $\hat{D}^{\dagger}$ to the inert core $|-\rangle:\left|S^{n_{s}} D^{n_{d}} I \eta\right\rangle=\left(\hat{S}^{\dagger}\right)^{n_{s}}\left(\hat{D}^{\dagger}\right)^{n_{d}}|-\rangle$, where $I$ is the total angular momentum, and $\eta$, an additional quantum number required to completely specify the states. The $n_{s}$ and $n_{d}$ are the numbers of $S$ and $D$ pairs, respectively. For a description of odd-nucleon systems, we add an unpaired nucleon in the $j$ orbital to the even-nucleon system. The state is now written as $\left|j S^{n_{s}} D^{n_{d}} I \eta\right\rangle=\left[\hat{c}_{j}^{\dagger}\left|S^{n_{s}} D^{n_{d}} I^{\prime} \eta\right\rangle\right]^{(I)}$, where $\hat{c}_{j}^{\dagger}$ represents the nucleon creation operator for the $j$ orbital. The odd-mass (neutron odd and proton even) nuclear state with total spin $I$ and its projection $M$ is written as a product of the above odd-neutron state and the even-proton state:

$$
|\Phi(I M \eta)\rangle=\left[\left|j_{n} S_{n}^{\bar{n}_{s}} D_{n}^{\bar{n}_{d}} I_{n} \eta_{n}\right\rangle \otimes\left|S_{p}^{n_{s}} D_{p}^{n_{d}} I_{p} \eta_{p}\right\rangle\right]_{M}^{(I)}
$$

where $2\left(\bar{n}_{s}+\bar{n}_{d}\right)+1$ and $2\left(n_{s}+n_{d}\right)$ are the numbers of valence neutron holes and proton particles, respectively.

As for single-particle levels, all the five $0 g_{7 / 2}, 1 d_{5 / 2}, 1 d_{3 / 2}, 0 h_{11 / 2}$, and $2 s_{1 / 2}$ orbitals in the major shell between the magic numbers 50 and 82 are taken into account for both neutrons and protons. The effective Hamiltonian consists of the monopole and quadrupole pairing plus quadrupole-quadrupole interactions:

$$
\hat{H}=\sum_{t=n, p}\left[\sum_{j m} \varepsilon_{j t} \hat{c}_{j m t}^{\dagger} \hat{c}_{j m t}-G_{0 t} \hat{P}_{t}^{\dagger(0)} \hat{P}_{t}^{(0)}-G_{2 t} \hat{P}_{t}^{\dagger(2)} \cdot \tilde{\hat{P}}_{t}^{(2)}-\kappa_{t}: \hat{Q}_{t} \cdot \hat{Q}_{t}:\right]-\kappa_{n p} \hat{Q}_{n} \cdot \hat{Q}_{p} .
$$

The Hamiltonian is diagonalized in terms of the many-body basis wave functions in Eq. (1) as $\hat{H}\left|I_{i}^{\pi}\right\rangle=$ $E_{i}^{\pi}\left|I_{i}^{\pi}\right\rangle$, where $\left|I_{i}^{\pi}\right\rangle$ is the normalized eigenvector for the $i$ th state with spin $I$ and parity $\pi$, and $E_{i}^{\pi}$ is the eigenenergy for the state $\left|I_{i}^{\pi}\right\rangle$. The details of the PTSM calculations are written in Ref. [8].

\subsection{Nuclear EDM from interactions violating $P$ and $T$ invariance}

The EDM operator due to charge asymmetry in a nucleus is written as $\hat{D}_{\mathrm{ch}}=\sum_{i=1}^{A} e_{i}\left(\mathbf{r}_{i}-\mathbf{R}\right)$. Here $A$ is mass number of a specific nucleus, and $e_{i}$ represents charge for the $i$ th nucleon. We take $e_{i}=0$ for a neutron and $e_{i}=e$ for a proton. The $\mathbf{r}_{i}$ and $\mathbf{R}$ represent positions of the $i$ th nucleon and the center of mass of the whole nucleus, respectively. In the present study, we use perturbation theory to obtain the EDM

$$
d_{\mathrm{ch}}=\sum_{k=1} \frac{\left\langle\frac{1}{2}_{1}^{+}\left|\hat{D}_{\mathrm{ch}, \mathrm{z}}\right| \frac{1}{2}_{k}^{-}\right\rangle\left\langle\frac{1}{2}_{k}^{-}\left|V_{\pi(I)}^{P T}\right| \frac{1}{2}_{1}^{+}\right\rangle}{E_{1}^{+}-E_{k}^{-}}+\text {c.c. }
$$

for the $1 / 2_{1}^{+}$states. In Eq. (3), $V_{\pi(I)}^{P T}$ represents the isoscalar $(I=0)$, isovector $(I=1)$, and isotensor $(I=2)$ interactions violating $P$ and $T$ invariance. The $\hat{D}_{\mathrm{ch}, \mathrm{z}}$ is the third coordinate component of $\hat{D}_{\mathrm{ch}}$. Here $\left|\frac{1_{2}^{+}}{2}\right\rangle$ represents the first state with spin $1 / 2$ and parity + and $\left|\frac{1^{-}}{2}\right\rangle$ represents the $k$ th state with spin $1 / 2$ and parity -. All these states have projection (third component of spin) $1 / 2 . V_{\pi(I)}^{P T}$ is written as [10-12]

$$
\begin{aligned}
V_{\pi(0)}^{P T} & =-\frac{1}{8 \pi} \frac{m_{\pi}^{2}}{M_{N}} \bar{g}_{\pi N N}^{(0)^{\prime}} g_{\pi N N}\left(\tau_{1} \cdot \tau_{2}\right)\left(\sigma_{1}-\sigma_{2}\right) \cdot \mathbf{r} f(r) \\
V_{\pi(1)}^{P T} & =-\frac{1}{16 \pi} \frac{m_{\pi}^{2}}{M_{N}} \bar{g}_{\pi N N}^{(1)^{\prime}} g_{\pi N N}\left[\left(\tau_{1 z}+\tau_{2 z}\right)\left(\sigma_{1}-\sigma_{2}\right)+\left(\tau_{1 z}-\tau_{2 z}\right)\left(\sigma_{1}+\sigma_{2}\right)\right] \cdot \mathbf{r} f(r) \\
V_{\pi(2)}^{P T} & =-\frac{1}{8 \pi} \frac{m_{\pi}^{2}}{M_{N}} \bar{g}_{\pi N N}^{(2)^{\prime}} g_{\pi N N}\left(3 \tau_{1 z} \tau_{2 z}-\tau_{1} \cdot \tau_{2}\right)\left(\sigma_{1}-\sigma_{2}\right) \cdot \mathbf{r} f(r) \\
f(r) & =\frac{\exp \left(-m_{\pi} r\right)}{m_{\pi} r^{2}}\left(1+\frac{1}{m_{\pi} r}\right)
\end{aligned}
$$


Here $m_{\pi}$ and $M_{N}$ are masses of pion and nucleon, respectively. $\tau_{i z}$ is the third component of isospin operator. $g_{\pi N N}$ is the strong $\pi N N$ coupling constant, and $\bar{g}_{\pi N N}^{(I)^{\prime}}$ is the strong $\pi N N$ constant violating $P$ and $T$ invariance for the isospin $I$ component. In the following we abbreviate $\bar{g}_{\pi N N}^{(I)^{\prime}}$ as $\bar{g}^{(I)}$ and $g_{\pi N N}$ as $g$.

\subsection{Nuclear EDM from intrinsic nucleon EDM}

The nuclear EDM operator arising from intrinsic nucleon EDM is written as $\hat{D}_{\text {int }}=\sum_{i=1}^{A} \hat{d}_{i}$, where the $i$ th nucleon intrinsic EDM operator $\hat{d}_{i}$ is expressed in the non-relativistic approximation as

$$
\hat{d}_{i}=\frac{1}{2}\left[\left(1-\tau_{i z}\right) d_{n} \hat{\sigma}_{i n}+\left(1+\tau_{i z}\right) d_{p} \hat{\sigma}_{i p}\right] .
$$

Here $d_{n}$ and $d_{p}$ are the intrinsic EDMs for a neutron and a proton, respectively, and $\hat{\sigma}_{i t}$ represents the spin operator. Then the intrinsic nuclear EDM is calculates as

$$
d_{\text {int }}=\left\langle\frac{1}{2}_{1}^{+}\left|\hat{D}_{\text {int, } \mathrm{z}}\right| \frac{1}{2}_{1}^{+}\right\rangle=\left\langle\hat{\sigma}_{n z}\right\rangle d_{n}+\left\langle\hat{\sigma}_{p z}\right\rangle d_{p},
$$

where projection of the state is taken as $M=1 / 2$.

\section{Numerical results}

\subsection{Nuclear EDM by interactions violating $P$ and $T$ invariance}

Firstly, we calculate nuclear EDMs for the $1 / 2_{1}^{+}$states in Xe isotopes arising from interactions which violate $P$ and $T$ invariance. Fig. 1 shows the results of the EDMs in Eq. (3). As seen from the figure, for most of the nuclei considered, isotensor components are the biggest among the three assuming $\bar{g}^{(0)}=\bar{g}^{(1)}=\bar{g}^{(2)}$.

If we consider only the isoscalar component of the interaction $\left(V_{\pi(0)}^{P T}\right)$ for the $1 / 2_{1}^{+}$state (the ground state) in ${ }^{129} \mathrm{Xe}$, we have

$$
\left|d_{\mathrm{ch}}\left({ }^{129} \mathrm{Xe}\right)\right|=0.70 \times 10^{-17} \bar{g}^{(0)} \mathrm{g} e \mathrm{~cm} .
$$

Using a relation between the isoscalar coupling $\bar{g}^{(0)}$ and the $C P$ violating phase $\bar{\theta}$ in the QCD Lagrangian $\bar{g}^{(0)}=0.027 \bar{\theta}[13]$, and adopting the standard value $g=13.5$, we have

$$
\left|d_{\mathrm{ch}}\left({ }^{129} \mathrm{Xe}\right)\right|=0.26 \times 10^{-17} \bar{\theta} e \mathrm{~cm} \text {. }
$$

Also, using the upper limit $\bar{\theta}=3 \times 10^{-10}$ obtained by the atomic ${ }^{199} \mathrm{Hg}$ experiment [5], the upper limit of the ${ }^{129} \mathrm{Xe}$ nuclear EDM is estimated as

$$
\left|d_{\mathrm{ch}}\left({ }^{129} \mathrm{Xe}\right)\right|<0.77 \times 10^{-27} e \mathrm{~cm} \text {. }
$$

\subsection{Nuclear EDM from intrinsic nucleon EDM}

Secondly, we calculate nuclear EDMs for the $1 / 2_{1}^{+}$states of Xe isotopes coming from the intrinsic nucleon EDM. Fig. 2 shows the quenching factors $\left\langle\hat{\sigma}_{t z}\right\rangle$ in Eq. (9). The factors for the protons $\left\langle\hat{\sigma}_{p z}\right\rangle$ are marginally small, but those for the neutrons $\left\langle\hat{\sigma}_{n z}\right\rangle$ increase as the neutron particle number. Using the observed upper limit for the neutron EDM $\left|d_{N}\right|<2.9 \times 10^{-26} e \mathrm{~cm}$, the upper limit of the EDM for the $1 / 2_{1}^{+}$state in ${ }^{129} \mathrm{Xe}$ EDM coming from the intrinsic nucleon EDM is predicted to be

$$
\left|d_{\text {int }}\left({ }^{129} \mathrm{Xe}\right)\right|<6.7 \times 10^{-27} \mathrm{ecm} .
$$




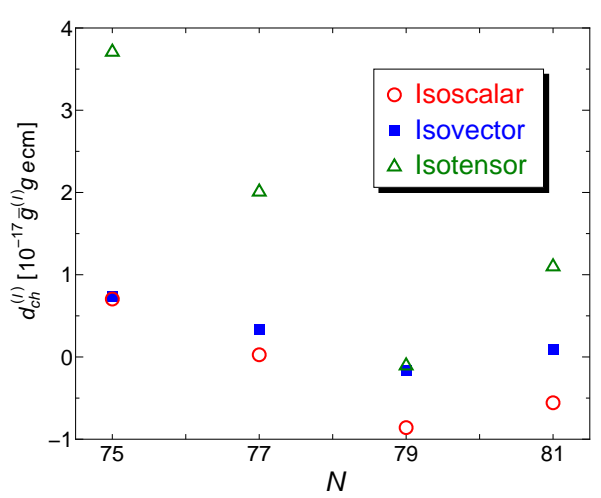

Figure 1. EDMs for $1 / 2_{1}^{+}$states in Xe isotopes from interactions violating $P$ and $T$ invariance in unit of $10^{-17} \bar{g}^{(I)} \mathrm{g} e \mathrm{~cm}(I=0,1,2)$. The circles, squares and triangles represent isoscalar, isovector and isotensor components, respectively.

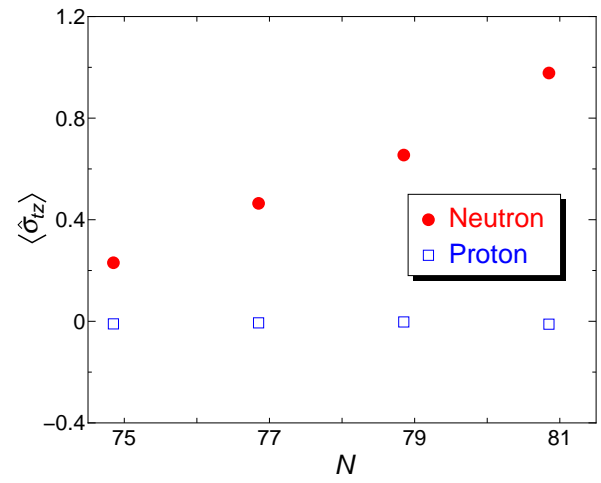

Figure 2. The factors $\left\langle\hat{\sigma}_{t z}\right\rangle(t=n$ or $p)$ for the Xe isotopes in $1 / 2_{1}^{+}$states. The circles and squares represent the values of $\left\langle\hat{\sigma}_{n z}\right\rangle$ and $\left\langle\hat{\sigma}_{p z}\right\rangle$, respectively.

\section{Summary}

We calculate nuclear EDMs of the $1 / 2_{1}^{+}$states in Xe isotopes in terms of the pair truncated shell model. Nuclear EDMs arise from two sources: one from interactions violating parity and time reversal invariance $\left(d_{\mathrm{ch}}\right)$ and the other from the intrinsic nucleon $\operatorname{EDM}\left(d_{\mathrm{int}}\right)$. We evaluate each upper limit of the EDM for the $1 / 2_{1}^{+}$state in ${ }^{129} \mathrm{Xe}$ from the two different sources.

\section{References}

[1] J. S. M. Ginges and V. V. Flambaum, Phys. Rep. 397, 63 (2004).

[2] C. A. Baker et al., Phys. Rev. Lett. 97, 131801 (2006).

[3] M. Pospelov and A. Rits, Ann. Phy. (NY), 318, 119 (2005).

[4] M. A. Rosenberry et al., Phys. Rev. Lett. 86, 22 (2001).

[5] W. C. Griffith et al., Phys. Rev. Lett. 102, 101601 (2009).

[6] S. Oshima, Phys. Rev. C 81, 038501 (2010).

[7] C. Itoi and S. Oshima, J. Phy. Soc. Jpn. 97, 103201 (2010).

[8] N. Yoshinaga et al., Phys. Rev. C 87, 044332 (2013).

[9] K. Higashiyama et al., to be published.

[10] W. C. Haxton, and E. M. Henley, Phys. Rev. Lett. 51, 1937 (1983).

[11] P. Herczeg, Hyperfine Interact. 43, 77 (1988).

[12] P. Herczeg, Hyperfine Interact. 75, 127 (1992).

[13] R. J. Crether et al., Phys. Lett. 88B, 123 (1979); 91B, 487 (1980). 